\title{
Sorafenib Combined with Radio-frequency Ablation Compared with Sorafenib Alone in Treatment of Hepatocellular Carcinoma Invading Portal Vein: A Western Randomized Controlled Trial
}

\author{
ANTONIO GIORGIO ${ }^{1,2}$, MARIA GABRIELLA MEROLA ${ }^{2}$, LUCA MONTESARCHIO ${ }^{1}$, \\ FRANCESCA MEROLA ${ }^{2}$, BRUNO SANTORO ${ }^{2}$, CARMINE COPPOLA $^{3}$, PIETRO GATTI ${ }^{4}$, \\ FERDINANDO AMENDOLA ${ }^{1}$, ANTONELLA DI SARNO ${ }^{5}$, ANDREA CALVANESE ${ }^{1}$, \\ PAOLO MATTEUCCI ${ }^{6}$ and VALENTINA GIORGIO ${ }^{7}$ \\ ${ }^{1}$ Interventional Ultrasound Unit, Tortorella Clinical Institute, Salerno, Italy; \\ ${ }^{2}$ Interventional Ultrasound Unit, Athena Cinical Institute, Caserta, Italy; \\ ${ }^{3}$ Hepatology Unit, Gragnano Hospital, Gragnano, Naples, Italy; \\ ${ }^{4}$ Internal Medicine Unit, Ostuni Hospital, Brindisi, Italy; \\ 5 Interventional Ultrasound Unit, D. Cotugno Hospital, Naples, Italy; \\ ${ }^{6}$ Radiotherapy and Oncology Department, Campus Biomedico University, Rome, Italy; \\ ${ }^{7}$ Pediatric Department, Fondazione Policlinico Gemelli, Rome, Italy
}

\begin{abstract}
Aim: To compare in a randomized controlled trial (RCT) 3-year survival of cirrhotic patients with hepatocellular carcinoma (HCC) accompanied by portal vein tumor thrombus $(P V T T)$ treated with sorafenib plus percutaneous radiofrequency ablation (RFA) of both intraparenchymal HCC and PVTT (combination Group) or sorafenib alone (sorafenibalone Group). Patients and Methods: Ninety-nine consecutive Child A cirrhotics were randomized to receive RFA of both HCC and main portal vein tumor thrombus (MPVTT) plus sorafenib $(n=49)$ or sorafenib alone $(n=50)$. Results: One-, 2- and 3-year survival rates were $60 \%, 35 \%$ and $26 \%$, respectively, in the combination group and $37 \%$ and $0 \%$ at 1 - and 2-year, respectively, in the sorafenib alone group. At multivariate analysis, the combination of RFA of both HCC and MPVTT was the only factor predicting survival. Conclusion: Use of RFA of both HCC and MPVTT plus sorafenib significantly increases 3year survival compared to sorafenib alone.
\end{abstract}

A. Giorgio was the former Director of Interventional US Unit of D.
Cotugno Hospital.

Correspondence to: Antonio Giorgio, MD, Viale Colli Aminei, 491, 80131, Naples, Italy. Tel: +393287711764, e-mail: agiorgio28@ gmail.com

Key Words: Hepatocellular carcinoma, portal vein tumor thrombus, liver cirrhosis, radiofrequency ablation, sorafenib.
Hepatocellular carcinoma (HCC) is the most common primary malignancy of the liver and the world's fifth most common cancer (1). The characteristic of this tumor is that it predominantly occurs in patients with liver cirrhosis and viral chronic infection by hepatitis B virus (HBV) and hepatitis $\mathrm{C}$ virus (HCV) (1). HCC has a great tendency to invade portal venous system and portal vein tumor thrombosis is reportedly found in $10 \%-40 \%$ of HCC patients at diagnosis (2-4). Portal vein tumor thrombus (PVTT) is a serious event; in fact, prognosis of patients with HCC and PVTT is poor if patients are left untreated; a median survival time of 2.7- 4.0 months is reported (5).

The main therapeutic option for these patients is treatment with sorafenib that is the first systemic targeted therapy showing significant survival benefits as it has shifted the HCC treatment paradigm. Results from pivotal trials demonstrated that sorafenib consistently increased overall survival in different patient populations across geographic regions and etiologies $(4,6)$.

At the end of the 1990s, radiofrequency ablation (RFA) was introduced in clinical practice (7); then, in the 2012, the European Association for the Study of the Liver (EASL) and American Association for the Study of Liver Diseases (AASLD) practice guidelines for HCC management, RFA was recommended as the main ablative therapy in cirrhotics in stage A of the Barcelona clinic liver cancer (BCLC) classification (3, 8). Today, percutaneous RFA ablation has no place in intermediate or advanced stage (i.e. portal venous invasion) of 
HCC disease in BCLC classification (8). Most recently, in patients with HCC and neoplastic main portal vein invasion, percutaneous RFA of both the nodule and the PVTT has been proposed (9). After the first report on safety and efficacy of this new ablative procedure (9), that was called by us RF Percutaneous Thrombectomy (RF-PTh), long-term results were also published with encouraging cumulative 5-year survival (10). Therefore, we thought to add percutaneous thrombectomy to sorafenib alone to verify if the combination of sorafenib plus RF-PTh was superior to sorafenib alone in treatment of HCC with main portal vein invasion in cirrhotic patients.

Thus, the aim of our study was to compare, in a randomized controlled trial (RCT), 3-year survival of cirrhotics with HCC and main portal vein tumor thrombus (MPVTT) treated with RFA of both HCC and MPVTT plus sorafenib (combination group) or sorafenib alone.

\section{Patients and Methods}

Patients. This study was approved by our Ethics Committee and an informed written consent was obtained from all patients. From June 2011 to July 2014, ninety-nine Child-Pugh A naïve cirrhotic patients consecutively seen in our Unit with HCC and MPVTT were randomly assigned to receive RFA of both HCC and MPVTT plus sorafenib ( $n=49$-combination group) or sorafenib alone $(n=50$ sorafenib alone group). There were 73 males and the patients' groups had a mean age of 71 year. The 128 HCC nodules of all patients ranged in diameter between 2.1 and $6.5 \mathrm{~cm}$. Cirrhosis was due to $\mathrm{HCV}$ in 59 patients, $\mathrm{HBV}$ in 33 patients and to unknown origin in 7 . None of the patients had ever been treated before and in all of the patients the presence of the nodule was seen for the first time during an occasionally ultrasound (US) abdominal examination because of their unknown liver cirrhosis.

Inclusion criteria were: i) Child-Pugh A 5-6 liver cirrhosis; ii) a single $\mathrm{HCC}$ nodule $<=6.5 \mathrm{~cm}$ in diameter and concomitant MPVTT and iii) maximum $3 \mathrm{HCC}$ nodules with the largest one no more than $5 \mathrm{~cm}$ with MPVTT.

Exclusion criteria: Patients with ascites, bilirubin $>2 \mathrm{mg} / \mathrm{dl}$ and with extrahepatic spread of disease.

Diagnosis of HCC nodules was made according to specific dynamic imaging findings on enhanced computed tomography (CT) and /or enhanced magnetic resonance imaging (MRI). Diagnosis of PVTT was made with fine-needle aspiration biopsy (FNAB) in all cases. Tables I and II report the main clinical, imaging and laboratory data of the patients studied. No statistically significant difference was present between the combination group and the sorafenib alone group.

The primary end point of the study was 3-year survival.

Randomization to RFA of MPVTT plus sorafenib and sorafenib alone was carried out prospectively with the use of a coded list compiled from a random number generator; the code was fully blinded to the field staff and trial participants were blinded to the code. The sample size was calculated considering $44 \% 3$-year survival rate in the control group (sorafenib alone) (6) and assuming that the experimental group (RFA-MPVTT plus sorafenib) would obtain more than a $20 \%$ increase in survival. An alpha error of 0.05 and a study power of about $80 \%$ (beta $=0.20$ ) were considered. The number of patients per arm was calculated to be more than 37 patients.
Percutaneous procedure. RFA-MPVTT was performed under unconscious sedation by the same physician (A.G.) who had more than 30 years' experience in interventional US. All percutaneous procedures were performed within 7 days of diagnosis. RFA of PVTT was performed as reported elsewhere (9). Briefly, RFA of MPVTT was performed under US guidance using a perfused electrode-needle, (caliber $15 \mathrm{G}$, exposed tip 1.5-2.0 $\mathrm{cm}$, according to the thrombus width) (HiTT, Integra; Nottinghen, Germanyand RF medical, Ltd., Seul, Korea), connected to an RF generator at a power of 80-100 Watt for 5-8 minutes: when the portal trunk appeared completely hyperechoic, the RFA application was considered sufficient and the electrode needle was withdrawn with the RF generator still on, so as to avoid seeding. RFA of the portal thrombus was carried out firstly on the thrombus in the portal vein, taking care to avoid hepatic artery and common bile duct and, then, on the HCC intrahepatic nodule(s) (9).The number of sessions for ablation of both HCC nodules and MPVTT was scheduled as follows: one session in case of HCC nodules up to $3 \mathrm{~cm}$ and MPVTT length no more than $1.5 \mathrm{~cm}$; two sessions in case of HCC nodules up to $5 \mathrm{~cm}$ and MPVTT length no more than $2.5 \mathrm{~cm} ; 3$ sessions in case of HCC nodules up to $6.5 \mathrm{~cm}$ and MPVTT length no more than $3.5 \mathrm{~cm}$. The day after percutaneous RFA procedures all patients underwent clinical and laboratory tests, as well as abdominal US.

After RF ablation HCC nodule's necrosis was evaluated using enhanced triphasic CT, while the recanalization of portal vessels was analyzed using color Doppler and CEUS (9).

Sorafenib was administered according to standard protocol, i.e. $800 \mathrm{mg} / \mathrm{die}$, per o.s. either in the combination or sorafenib alone groups. Sorafenib treatment started soon after the end of scheduled RFA sessions of both HCC and PVTT and soon after diagnosis of HCC and PVTT in the sorafenib alone group.

Follow-up. All patients underwent post-treatment follow-up, starting from the first day after the procedure through clinical evaluation and laboratory exams, abdominal US/Color Doppler/CEUS; then, every week, for the first 4 weeks, all patients underwent abdominal US/Color-Doppler evaluation. During the following 36 months, abdominal US/Color Doppler/CEUS and CT scan evaluation were made monthly, whereas abdominal US and serum assay of alphafetoprotein (AFP) were made every 2 months and CEUS and CT scan every 6 months (9).

Statistical analysis. Quantitative and qualitative variables were calculated according to Student's $t$-test and Chi-square test, respectively. Survival was calculated using the Kaplan-Meier method and the differences between the two curves were calculated with the log-rank test.

Univariate and multivariate analyses were carried out using the Cox proportional hazards models. Results are presented as hazard ratios (HRs) with corresponding 95\% confidence intervals (CI) and $p$-values. A $p$-value less than 0.05 was considered as being statistically significant.

\section{Results}

The follow-up ranged from 6 to 42 months. The range of RFA sessions was 1-3 with a mean of 1.9.

One-, 2- and 3-year cumulative survival rates in the combination group were $60 \%, 35 \%$ and $26 \%$, respectively, 


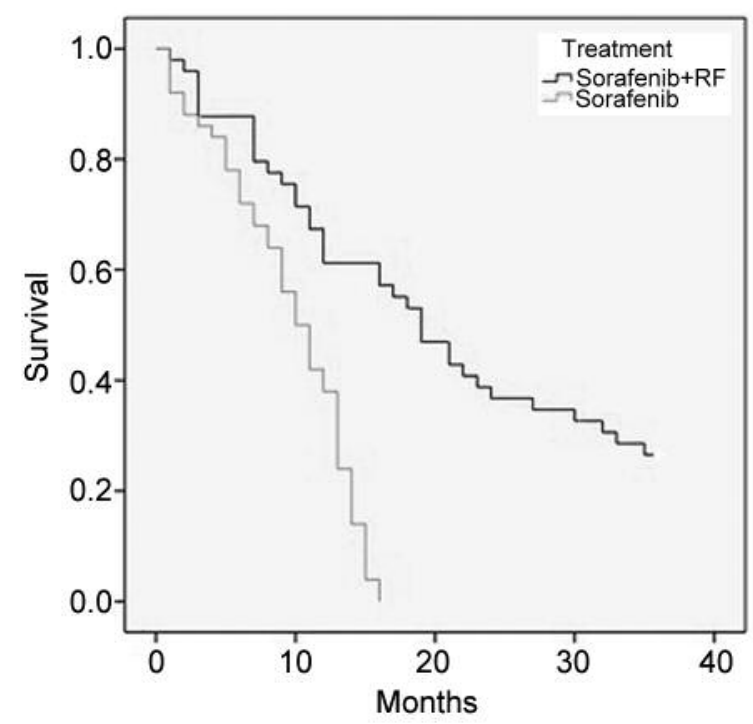

Figure 1. Cumulative survival rates of combination group and Sorafenib alone.

while 1- and 2-year survival rates in the sorafenib group alone were $37 \%$ and $0 \%$, respectively, with a significant statistical difference $(\mathrm{HR}=2.87 ; 95 \% \mathrm{CI}=1.61-5.39, p<0.001)$, Figure 1 .

At univariate analysis, combination of sorafenib and percutaneous ablation of $\mathrm{HCC}$ and MPVTT $(\mathrm{HR}=2.36$; $95 \% \mathrm{CI}=28-4-03, p<0.002)$, number of HCC nodules $(\mathrm{HR}=1.67$; $95 \% \mathrm{CI}=060-4.91, p=0.02)$ and length of MPVTT $(\mathrm{HR}=1.65$; $95 \% \mathrm{CI}=0.59-4.87, p=0.02$ ) were independent risk factors for survival; at multivariate analysis, only combined use of sorafenib and percuraneous thrombectomy was the independent predictor of survival $(\mathrm{HR}=2.89 ; 95 \% \mathrm{CI}=1.55-5.43, p<0.001)$.

Complications and adverse events in the 2 groups. Combination group: Complications due to RFA of MPVTT: no case of death was observed in the combination group due to interventional RFA procedure. One case of hemoperitoneum was observed, spontaneously healed. Five patients presented with ascites after RFA, treated with diuretics. In 4 patients, increased transaminases (resolved within one week) were observed.

Adverse events after addition of sorafenib: abdominal pain was observed in 7 patients; diarrhea in 6; asthenia in 11; weight loss in 10 and hand and foot syndrome in 5 patients, respectively. One patient developed acute pancreatitis after two days of adding sorafenib. Ten patients (25\%) dropped out.

Sorafenib alone group: In the sorafenib alone group adverse events were observed in 16 (39\%) cases: abdominal pain in 7 , diarrhea in 6 , weight loss in 8 , asthenia in 8 , hand foot syndrome in 4 . The drop-out in this group was $29 \%$ (12 patients).
Table I. Baseline patient's characteristics.

\begin{tabular}{lcc}
\hline & Combination group & Sorafenib alone \\
\hline Number of patients & 49 pts & 50 pts \\
Mean age & 71 (range=69-75) & 72 (range $=70-76)$ \\
Gender (males) & 37 & 36 \\
Follow-up (months) & $6-42$ & $5-41$ \\
Child-Pugh & & \\
A5 & 25 & 26 \\
A6 & 24 & 24 \\
HBV & 16 & 17 \\
HCV & 32 & 27 \\
Unknown origin & 4 & 3 \\
Number of nodules & 63 & 65 \\
Diameter range & $2.3-6.5 \mathrm{~cm}($ mean $=4.4)$ & $2.1-6.5 \mathrm{~cm}$ (mean=4.3) \\
\hline
\end{tabular}

pts, Patients; HBV, hepatitis B virus; HCV, hepatitis C virus.

Table II. Baseline clinical and laboratory variables.

\begin{tabular}{lcc}
\hline & Combination group & Sorafenib alone \\
\hline Albumin $(\mathrm{g} / \mathrm{dl})$ & $3.33 \pm 0.91$ & $3.37 \pm 0.82$ \\
Bilirubin $(\mathrm{mg} / \mathrm{dl})$ & $0.94 \pm 0.30$ & $0.88 \pm 0,44$ \\
International normalized ratio & $1.04 \pm 0.2$ & $1,03 \pm 0,13$ \\
Platelets $\left(\times 1,000 / \mathrm{mm}^{3}\right)$ & $115.05 \pm 7.31$ & $116.50 \pm 10,30$ \\
Alfa-fetoprotein $(\mathrm{mg} / \mathrm{ml})$ & $83.01 \pm 89.056$ & $80.05 \pm 2$ \\
Ascites \% & 0 & 0 \\
\hline
\end{tabular}

\section{Discussion}

Up to now, sorafenib is the first-line treatment of choice for advanced HCC. However, the benefits of this anti-angiogenic drug in HCC patients with portal vein tumor thrombosis remain uncertain (11).

Although sorafenib is regarded as the standard treatment option for advanced $\mathrm{HCC}$, its clinical efficacy in $\mathrm{HCC}$ patients with PVTT has been frequently challenged by other interventions, such as hepatic arterial infusion chemotherapy (HAIC), transarterial chemoembolization (TACE), radioembolization, radiotherapy and hepatic resection. Our aim was to explore, in a RCT, whether or not the addition of RFA could improve the outcomes of sorafenib in HCC patients with MPVTT and Child-Pugh class A.

In fact, our study focused on the management of 99 ChildPugh A naïve consecutive cirrhotic patients with HCC and MPVTT (single HCC $\leq 6.5 \mathrm{~cm}$ and PVTT or maximum 3 HCC nodules no more than $5 \mathrm{~cm}$ with PVTT) comparing overall 3-year survival of the combination treatment by RFA of both HCC and PVTT plus sorafenib in 49 patients to sorafenib alone in 50 patients. 
Our data suggest that RFA of both HCC and MPVTT represents a significant factor for increased survival in addition to sorafenib alone in cirrhotics having an advanced disease, such as HCC main portal vein invasion. In fact, in our study, 3-year cumulative survival was significantly different between patients treated with RFA plus sorafenib in respect to patients treated with sorafenib alone. Patients with MPVTT had no probability to survive after 2 years when treated with sorafenib alone, while the probability to have 3-year survival was observed in $26 \%$ of patients with MPVTT when RF percutaneous thrombectomy was added to sorafenib after RFA of both HCC and PVTT.

Up to now, to our knowledge, our study is the second RCT published as full text in the literature comparing sorafenib alone with sorafenib plus other interventions. Some retrospective studies have shown that HAIC, hepatic resection and three-dimensional conformal radiotherapy might be superior to sorafenib in improving the overall survival. (11) In a multi-centre retrospective study conducted with 110 cases, HAIC was compared with sorafenib: the HAIC group achieved a longer overall survival time (median=7.1 months $v s .5 .5$ months, $p=0.011$ ). However, the multivariate analysis did not identify HAIC as a significant prognostic factor.

A Korean retrospective study compared the results of sorafenib with hepatic resection in 104 cases (12). The survival rate was higher in the hepatic resection group than in the sorafenib group (1-year rate $=63.6 \% v s .32 .3 \%$; 2-year rate $=31.3 \%$ vs. $5.6 \%$ ) but the HCC patients had PVTT located within the segmental branches and/or right and left portal vein and not in MPV as in our study.

Recently, similarly to our study, Yang and coworkers evaluated in a RCT the clinical benefit of combination of sorafenib with cryotherapy as an adjunctive treatment modality in HCC patients with PVTT receiving sorafenib (13). The combination provided a significantly longer overall survival time (median=12.5 months $v s .8 .6$ months). As in our study, multivariate Cox regression analysis identified combined use of sorafenib and cryotherapy as an independent predictor of survival.

\section{Conclusion}

Combined use of RFA of both HCC nodule and MPVTT plus sorafenib is much better than the use of sorafenib alone in cirrhotics with invasion of main portal trunk. Results of our RCT clearly indicate that 3-year survival of such kind of patients can be increased adding the alleged percutaneous thrombectomy to sorafenib alone in a safe and effective manner.

Because percutaneous thrombectomy should be performed by experienced operators, it is difficult to spread our procedure in a larger number of interventional ultrasound centers. Up to now, to our knowledge, only few Asian Centers have reported percutaneous RFA of MPVTT with results similar to ours. It is, thus, desirable that other Western Centers can reproduce these results in the near future.

\section{Disclosure}

All Authors read and approved the final manuscript. The Authors do not have any conflict of interest in connection with the submitted manuscript.

\section{References}

1 Ferlay J, Shin HR, Bray F, Forman D, Mathers C and Parkin DM: Estimates of worldwide burden of cancer in 2008: GLOBOCAN 2008. Int J Cancer 127: 2893-2917, 2010.

2 Tandon $P$ and Garcia-Tsao G: Prognostic indicators in hepatocellular carcinoma: A systematic review of 72 studies. Liver Int 29: 502-510, 2009.

3 European Association For The Study Of The Liver. European Organisation for Research and Treatment of Cancer. EASLEORTC clinical practice guidelines: management of hepatocellular carcinoma. J Hepatol 56: 908-943, 2012.

4 Llovet JM, Ricci S, Mazzaferro V,Hilgard P, Gane E, Blanc JF, de Oliveira AC, Santoro A, Raoul JL, Forner A, Schwartz M, Porta C, Zeuzem S, Bolondi L,Greten TF, Galle PR, Seitz JF, Borbath I, Häussinger D, Giannaris T, Shan M, Moscovici M, Voliotis D and Bruix J: Sorafenib in advanced hepatocellular carcinoma. N Engl J Med 359: 378-390, 2008.

5 Minagawa $\mathrm{M}$ and Makuuchi $\mathrm{M}$ : Treatment of hepatocellular carcinoma accompanied by portal vein tumor thrombus. World J Gastroenterol 12: 7561-7567, 2006.

6 Cheng AL, Kang YK, Chen Z, Tsao CJ, Qin S, Kim JS, Luo R, Feng J, Ye S, Yang TS, Xu J, Sun Y, Liang H, Liu J, Wang J, Tak WY, Pan H, Burock K, Zou J,Voliotis D and Guan Z: Efficacy and safety of sorafenib in patients in the Asia-Pacific region with advanced hepatocellular carcinoma: A phase III randomised, double-blind, placebo-controlled trial. Lancet Oncol 10: 25-34, 2009.

7 Rossi S, Ravetta V, Rosa L,Ghittoni G, Viera FT, Garbagnati F, Silini EM, Dionigi P, Calliada F, Quaretti P and Tinelli C: Repeated radiofrequency ablation for management of patients with cirrhosis with small hepatocellular carcinomas: A long-term cohort study. Hepatology 53: 136-147, 2011.

8 Bruix J and Sherman M: American Association for the Study of Liver Diseases. Management of hepatocellular carcinoma: An update. Hepatology 53: 1020-1022, 2011.

9 Giorgio A, Di Sarno A, de Stefano G, Farella N, Scognamiglio U, de Stefano M and Giorgio V: Hepatocellular carcinoma with cirrhosis: are patients with neoplastic main portal vein invasion eligible for percutaneous radiofrequency ablation of both the nodule and the portal venous tumor thrombus? Am J Roentgenol 193: 948-954, 2009.

10 Giorgio A, Di Sarno A, De Stefano G, Scognamiglio U, Farella N, Mariniello A, Esposito V, Coppola C and Giorgio V: Percutaneous radiofrequency ablation of hepatocellular carcinoma compared to percutaneous ethanol injection in treatment of cirrhotic patients: an Italian randomized controlled trial. Anticancer Res 31: 2291-2295, 2011. 
11 Qi X and Guo X: Sorafenib for the treatment of hepatocellula carcinoma with portal vein tumour thrombosis: a systematic review of comparative studies. Prz Gastroenterol 10: 142-147, 2015.

12 Lee JM, Jang BK, Lee YJ, Choi WY, Choi SM, Chung WJ, Hwang JS, Kang KJ, Kim YH, Chauhan AK, Park SY, Tak WY, Kweon YO, Kim BS and Lee CH: Survival outcomes of hepatic resection compared with transarterial chemoembolization or sorafenib for hepatocellular carcinoma with portal vein tumor thrombosis. Clin Mol Hepatol 22: 160-167, 2016.
13 Yang Y, Lu Y, Wang C, Bai W, Qu J, Chen Y, Chang X, An L, Zhou L, Zeng Z and Lou Mand Lv J: Cryotherapy is associated with improved clinical outcomes of sorafenib for the treatment of advanced hepatocellular carcinoma. Exp Ther Med 3: 171$180,2012$.

Received August 12, 2016

Revised September 6, 2016

Accepted September 7, 2016 